Supporting Information for

\title{
Collector Droplet Behavior During Formation of Nanowire
}

\author{
Junctions \\ Yanming Wang ${ }^{1}$, Tomáš Šikola, ${ }^{2,3}$, Miroslav Kolibal ${ }^{2,3 *}$
}

${ }^{1}$ Department of Materials Science and Engineering, Massachusetts Institute of Technology, 77 Massachusetts Ave, Cambridge, MA 02139, USA

${ }^{2}$ Institute of Physical Engineering, Brno University of Technology, Technická 2, 61669 Brno, Czech Republic

${ }^{3}$ CEITEC BUT, Brno University of Technology, Purkyňova 123, 61200 Brno, Czech Republic
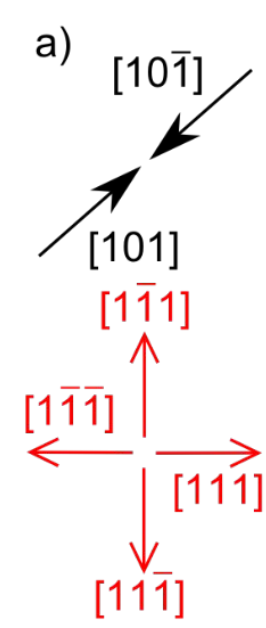
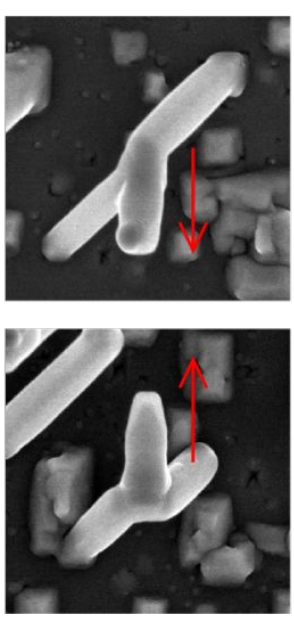
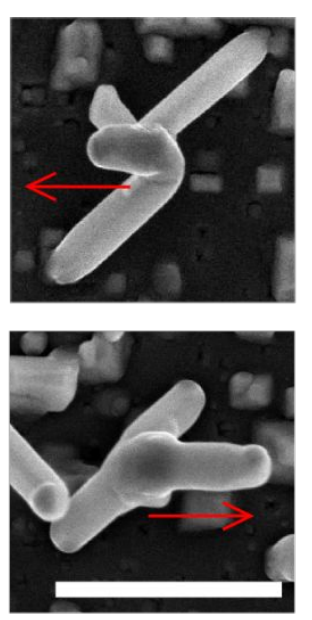
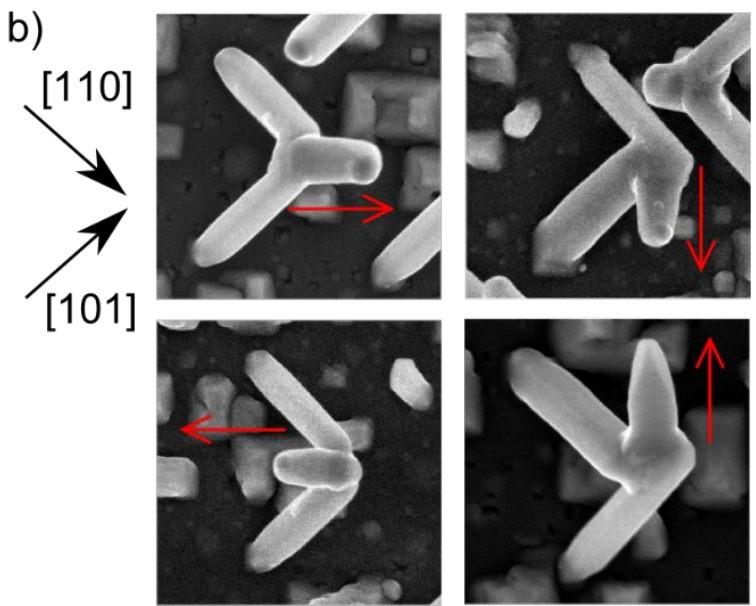

Fig. S1: All the possible nanowire growth directions after merging are shown (the same sample as shown in Fig. Ia in the main text). The images clearly demonstrate that the nanowire growth direction after the collision is independent of the initial geometry (all $<111>$ growth directions are possible if nanowires meet head-on (a) or inclined by $90^{\circ}$ (as viewed from top, b)). The black arrows indicate the initial $<110>$ growth directions of the nanowires (top view), the red arrows mark the $<111>$ directions after merging. The scale bar is the same for all images, $1 \mu$ m. 


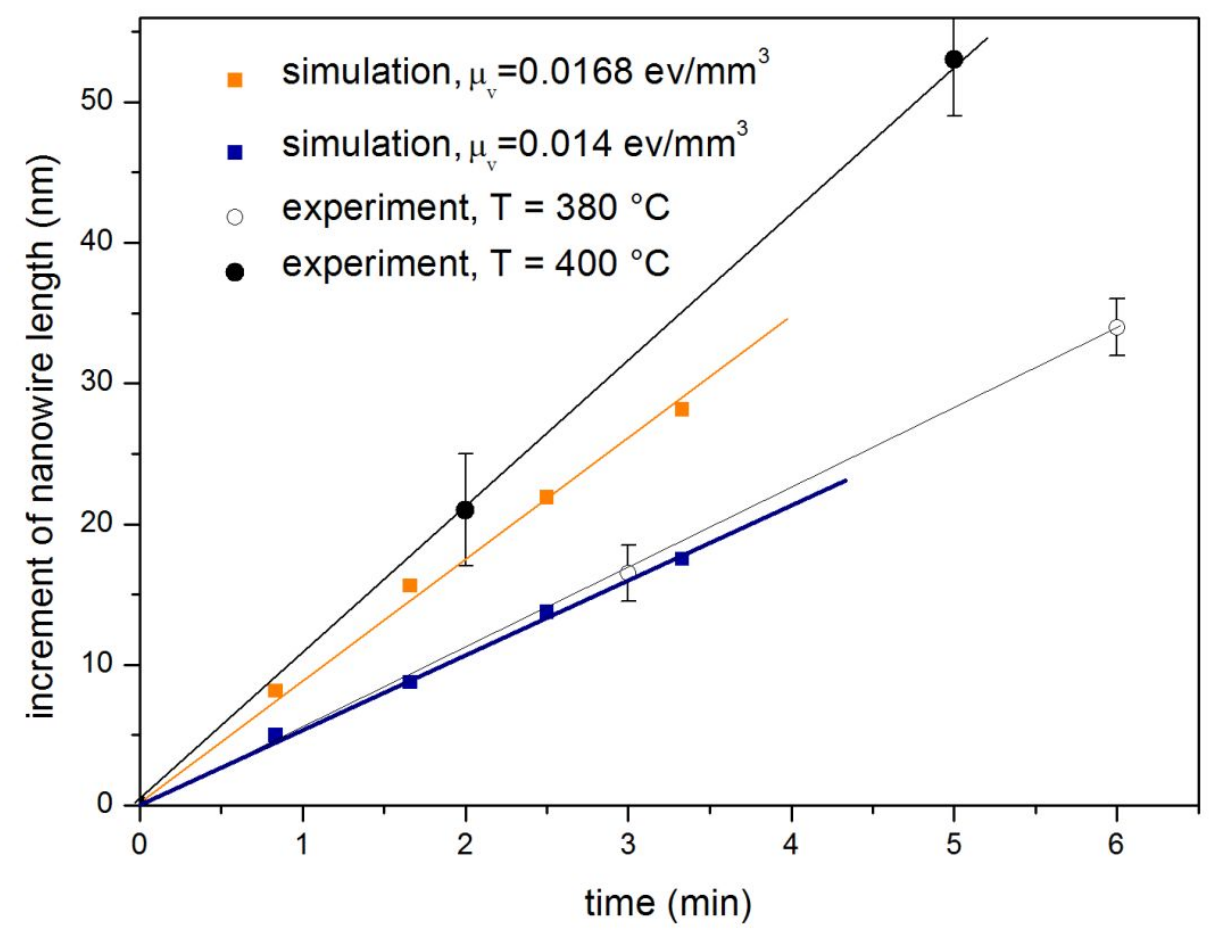

Fig. S2: The increment of nanowire length is plotted as a function of time for both phase field simulations (squares) with a vapor chemical potential density $\mu_{V}$ of $0.014 \mathrm{eV}^{\mathrm{nm}} \mathrm{m}^{3}$ (slow growth) and $0.0168 \mathrm{eV} / \mathrm{nm}^{3}$ (fast growth), respectively, and experiments (circles). The experimental data points were derived from real-time image sequences of nanowire growth at respective temperatures. The error bars are based on uncertainties related to the pixel resolution of SEM images in each sequence (not shown). 
a)
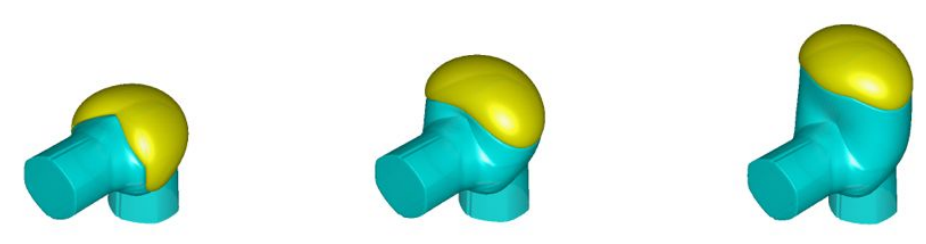

[110]
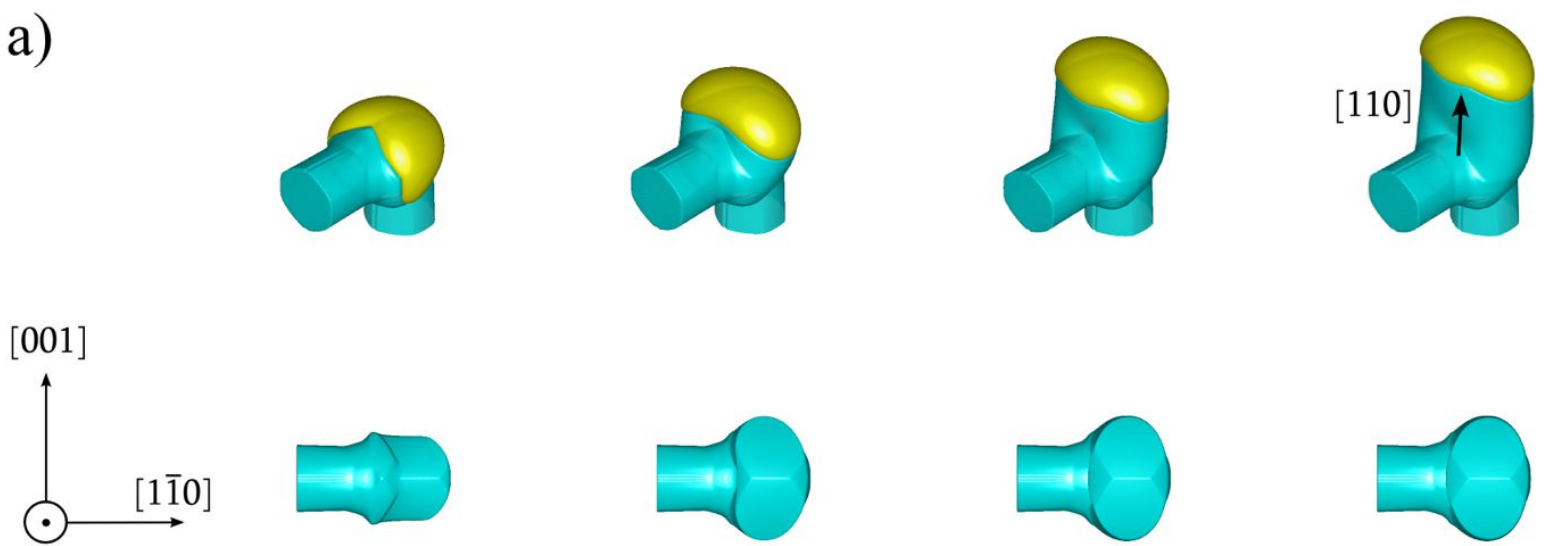

[110]

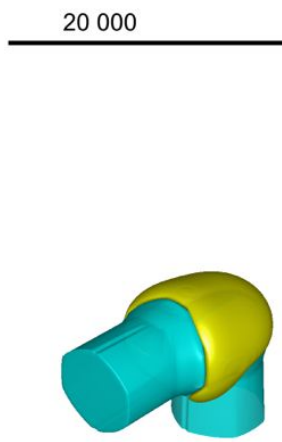

100000

200000

260000

simulation steps

b)

[11̄0]
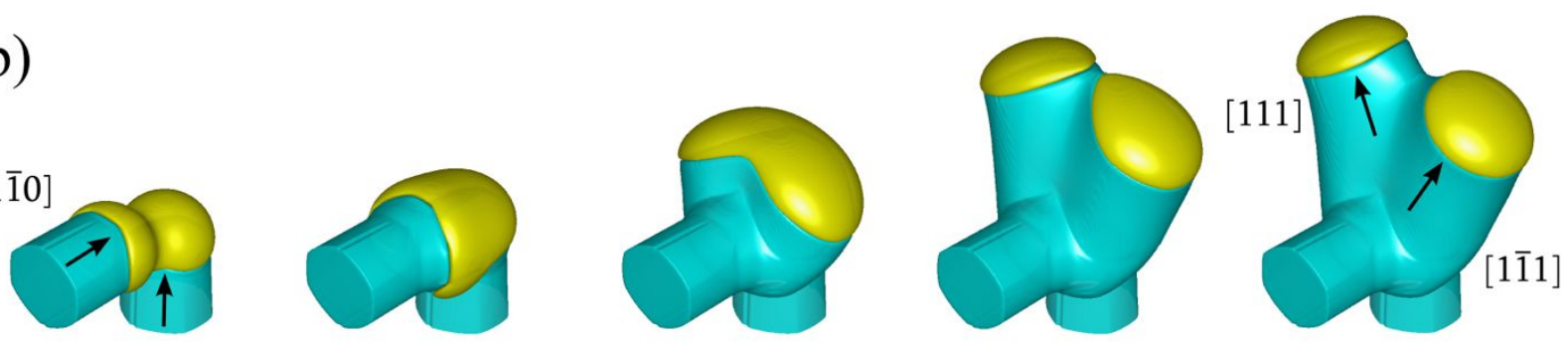

[110]
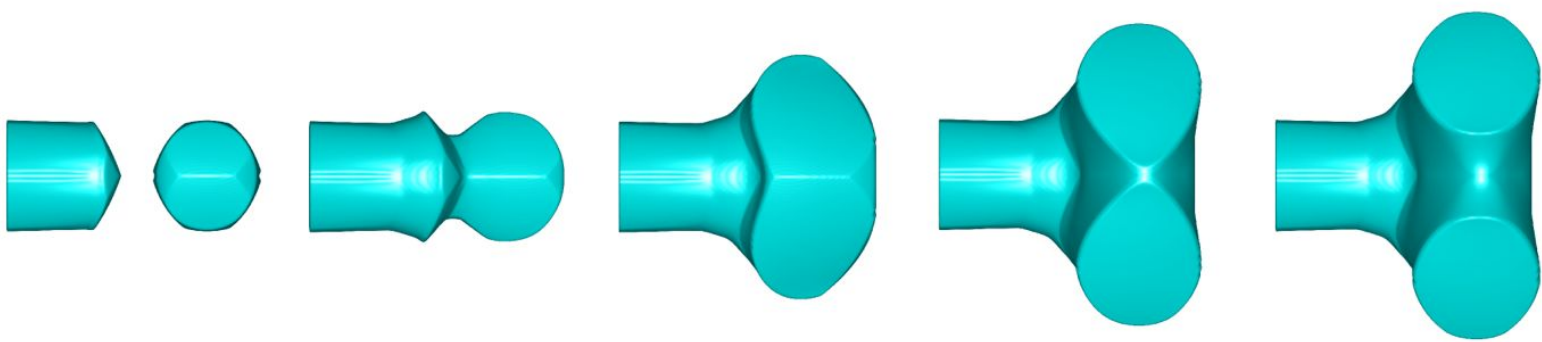

5000

50000

100000

150000

200000

simulation steps

Fig. S3: Phase field simulations of nanowire junction formation at different growth rate; the nanowires are perfectly aligned. a) At the slow growth rate $(\sim 5.3 \mathrm{~nm} / \mathrm{min})$, the droplets catalyzing growth of the initial $<110>$-oriented nanowires are merged into one, the " $Y$ " junction is formed and the nanowire grows in the $<110>$ direction, in contrast to the case of misaligned nanowires shown in Fig. 2a. b) At higher growth rate ( $8.7 \mathrm{~nm} / \mathrm{min})$, the merged droplet splits and the " $X$ " junction is formed. The growth orientation of newly formed nanowires is changed to 
$<111>$, similar to Fig. 2b. In both a) and b) the top row is tilted side view, bottom row is top view, including the coordination system. The evolution of the liquid-solid interface shape at the early stage is provided in Fig. S4.
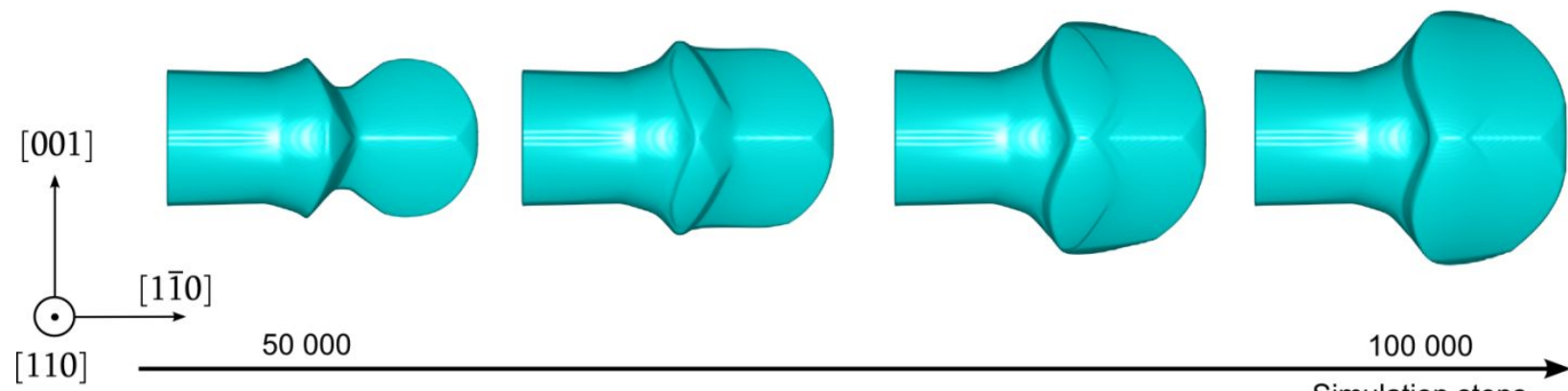

100000

Simulation steps

Fig. S4: Phase field simulation of the collision event shown in Fig. S3b (higher growth rate), here showing in detail the shape change of the liquid-solid interface at the early stage of the simulation before splitting. The droplet is removed to expose the liquid-solid interface more clearly. 


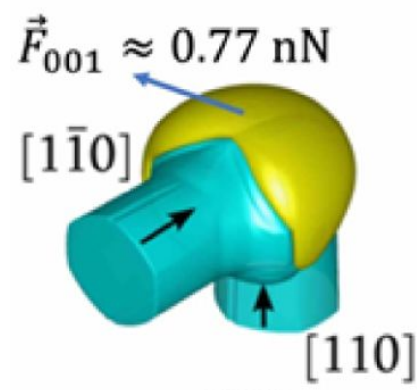

50000

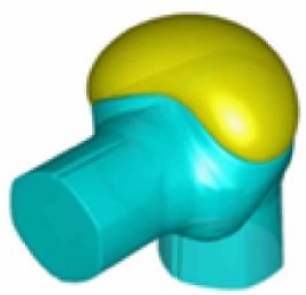

100000

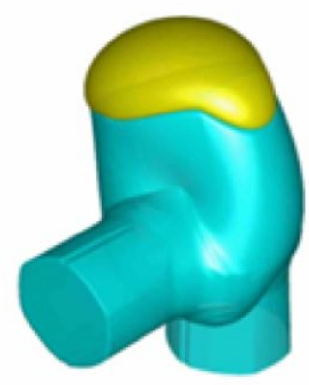

150000

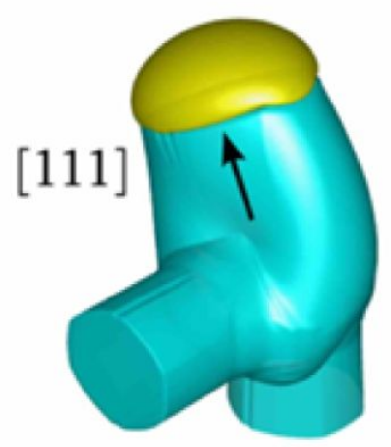

200000

simulation steps

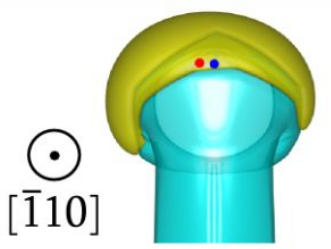

Fig. S5: Kinking towards $<111>$ direction after droplets' merging, induced by introducing a perturbation force of $0.77 \mathrm{nN}$ along the [001] direction to push the droplet out of symmetric position (at steps 0-5000). Similarly to the results shown in Fig. 2a, the liquid-solid growth interface changes towards an asymmetric shape, finally being formed by a single (111) plane. The droplet assymetry is documented by the view in [110] direction, showing the calculated center of mass marked by blue dot (symmetric position before the force is applied) and red dot (assymetric position, after the application of the force). The reasoning behind the external force could be e.g. shadowing of the diffusion flux as the wires get close to each other or temperature fluctuations as the wires merge. ${ }^{35}$ 
Phase Field Model Parameters. For $i, j=L, S, V$, the parameters $U_{i}$ and $\epsilon_{i}(\mathbf{n})$ (as a function of $\epsilon_{i 0}$, $\left.\epsilon_{i 1}, \epsilon_{i 2}, \epsilon_{i 3}\right)$ together determine the interfacial energies $\sigma_{i j}$ and the interface thickness $\xi_{i j}$. Explicitly, $\sigma_{i j}=\frac{1}{3} \sqrt{\left(U_{i}+U_{j}\right) \cdot\left[\epsilon_{i}^{2}(\mathbf{n})+\epsilon_{j}^{2}(\mathbf{n})\right]}, \quad \xi_{i j}=2 \sqrt{\left[\epsilon_{i}^{2}(\mathbf{n})+\epsilon_{j}^{2}(\mathbf{n})\right] /\left(U_{i}+U_{j}\right)}, \quad$ and $\quad \epsilon_{i}(\mathbf{n})=\epsilon_{i 0}$ $\left[1+\epsilon_{i 1} \cdot\left(n_{x}^{2} n_{y}^{2}+n_{y}^{2} n_{z}^{2}+n_{z}^{2} n_{x}^{2}\right)+\epsilon_{i 2} \cdot\left(n_{x}^{2} n_{y}^{2} n_{z}^{2}\right)+\epsilon_{i 3} \cdot\left(n_{x}^{2} n_{y}^{2}+n_{y}^{2} n_{z}^{2}+n_{z}^{2} n_{x}^{2}\right)^{2}\right]$, where $n_{x}, n_{y}$ and $n_{z}$ are the $x, y$ and $z$ components of the surface normal of the phase field local orientation. In Eq. $1, \mu_{i}$ is the chemical potential density for phase $i$, and $C\left(\phi_{i}\right)$ is a function of $\phi_{i}$ with a range of $[0,1]$ and a zero derivative at $\phi_{i}=0$ or 1 (one choice of $C\left(\phi_{i}\right)$ that satisfies the above requirements is $\left.C\left(\phi_{i}\right)=\left[\tanh \left(10 \phi_{i}-5\right)+1\right] / 2\right) \cdot \alpha$ specifies the direction and the magnitude of the external force perturbation on the liquid droplet, and $P$ gives the strength of the penalty on the unphysical three-phase mixing. Tab. S1 lists the values of the key model parameters. Besides the ones discussed above, $h$ is the grid size, $K_{S V}^{G L}$ is the kinetic coefficient at the liquid-vapor interface and $K_{0}^{G L}$ is the kinetic coefficient for other types of interfaces.

Tab. S1: Parameters of the multi-phase field model. $\boldsymbol{U}_{\boldsymbol{i}}$ and $P$ are in unit of $\mathrm{eV} / \mathrm{nm}^{3} . \boldsymbol{\epsilon}_{\boldsymbol{i} \mathbf{0}}^{2}$ is in unit of $\mathrm{eV} / \mathrm{nm}$. $h$ is in unit of $\mathrm{nm}$.

\begin{tabular}{ccccccccc}
\hline $\boldsymbol{U}_{\boldsymbol{L}}$ & $\boldsymbol{U}_{\boldsymbol{S}}$ & $\boldsymbol{U}_{\boldsymbol{V}}$ & $\boldsymbol{\epsilon}_{\mathbf{L 0}}^{2}$ & $\boldsymbol{\epsilon}_{\boldsymbol{S} \mathbf{0}}^{2}$ & $\boldsymbol{\epsilon}_{\boldsymbol{V} \mathbf{0}}^{2}$ & $\boldsymbol{h}$ & $\boldsymbol{\epsilon}_{\mathbf{L 1}}$ & $\boldsymbol{\epsilon}_{\boldsymbol{S 1}}$ \\
\hline 0.0134 & 0.0210 & 0.0454 & 0.0920 & 0.2805 & 0.4370 & 2.5 & -0.608 & 0.692 \\
\hline $\boldsymbol{\epsilon}_{\boldsymbol{V} \mathbf{1}}$ & $\epsilon_{L 2}$ & $\epsilon_{S 2}$ & $\epsilon_{V 2}$ & $\epsilon_{L 3}$ & $\epsilon_{S 3}$ & $\epsilon_{V 3}$ & $K_{S V}^{G L} / K_{0}^{G L}$ & $P$ \\
\hline $\mathbf{0 . 1 3 5}$ & 7.184 & -7.482 & -1.502 & 0.758 & -1.202 & -0.207 & $10^{-8}$ & 0.5 \\
\hline
\end{tabular}

